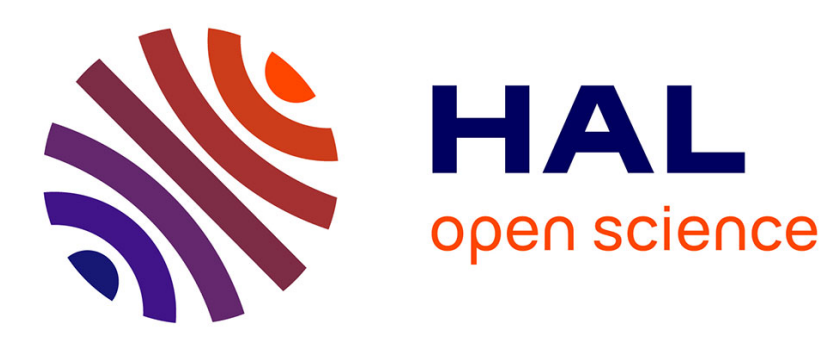

\title{
Dispersion dans un écoulement de Stokes
}

Christian Baudet, E. Guyon, Y. Pomeau

\section{To cite this version:}

Christian Baudet, E. Guyon, Y. Pomeau. Dispersion dans un écoulement de Stokes. Journal de Physique Lettres, 1985, 46 (21), pp.991-998. 10.1051/jphyslet:019850046021099100 . jpa-00232933

\section{HAL Id: jpa-00232933 https://hal.science/jpa-00232933}

Submitted on 1 Jan 1985

HAL is a multi-disciplinary open access archive for the deposit and dissemination of scientific research documents, whether they are published or not. The documents may come from teaching and research institutions in France or abroad, or from public or private research centers.
L'archive ouverte pluridisciplinaire HAL, est destinée au dépôt et à la diffusion de documents scientifiques de niveau recherche, publiés ou non, émanant des établissements d'enseignement et de recherche français ou étrangers, des laboratoires publics ou privés. 
Classification

Physics Abstracts

$47.55 \mathrm{~K}-47.55 \mathrm{M}-51.20$

\title{
Dispersion dans un écoulement de Stokes ( $\left.{ }^{*}\right)$
}

\author{
C. Baudet, E. Guyon
}

L.H.M.P., E.S.P.C.I., 10, rue Vauquelin, 75231 Paris Cedex 05, France

et Y. Pomeau

Service de Physique Théorique, C.E.N. Saclay, 91191 Gif sur Yvette, France

(Reçu le 17 juillet 1985, accepté le 17 septembre 1985)

\begin{abstract}
Résumé. - Nous décrivons des effets singuliers de la dispersion géométrique dans un milieu poreux granulaire à des grands nombres de Peclet en considérant d'abord la distribution des temps de résidence autour d'une seule sphère placée dans un champ de vitesse uniforme $U$. Il en résulte une singularité logarithmique de la dispersion en $U$ Log $U$ qui est due à l'écoulement lent au voisinage des points de stagnation de l'écoulement. Cette caractéristique est indépendante de l'écoulement loin des points de stagnation. Nous discutons l'application de ce résultat à un ensemble dilué de sphères (lit fixe).
\end{abstract}

\begin{abstract}
We approach the geometric dispersion effects in a granular porous medium at large Peclet numbers by first considering the distribution of residence times around a single sphere in a uniform applied velocity field $U$. We get a logarithmic singularity of the dispersion in $U \log U$ which is due to the slow flow near the stagnation points of the flow field. This feature is independent of the flow structure at large distances from the stagnation points. Thus the result can be applied to a random dilute array of spheres (fixed bed).
\end{abstract}

Nous nous intéressons au comportement d'un traceur inerte en solution dans un liquide qui s'écoule en régime permanent à travers un milieu poreux (effet de dispersion). Celui-ci est supposé consister en un assemblage de grains sphériques et nous considérons que ceux-ci sont suffisamment séparés pour pouvoir étudier, dans un premier temps, le régime d'écoulement autour d'une sphère unique (lit fixe dilué). Nous supposons aussi que l'écoulement est assez lent pour que le régime d'écoulement de Stokes aux faibles nombres de Reynolds $(R e)$ s'applique.

Les effets combinés de la diffusion moléculaire (diffusivité $D_{m}$ ) et de la convection par l'écoulement donnent lieu au phénomène de dispersion (par opposition à la diffusion observable en l'absence d'écoulements).

$\left(^{*}\right)$ Une première discussion de ce problème a été présentée par « le groupe poreux P.C. » à la rencontre N.A.T.O. « Scaling effects in disordered matter » (1985); à paraître, Plenum Press (1985). 
Dans les cas les plus favorables (e.g. chromatographie), on peut décrire la dispersion par une équation macroscopique de la forme $[1,2]$ :

$$
\frac{\partial c}{\partial t}+|\mathbf{U}| \frac{\partial c}{\partial x_{\|}}=D_{\|} \frac{\partial^{2} c}{\partial x_{\|}^{2}}+D_{\perp} \frac{\partial^{2} c}{\partial x_{\perp}^{2}}
$$

où $c$ et $\mathbf{U}$ sont des moyennes spatiales de la concentration et de la vitesse sur un élément de volume représentatif de la structure du milieu poreux ; $D_{\|}$et $D_{\perp}$ sont des coefficients phénoménologiques décrivant l'étalement d'une tache de traceur dans les directions parallèle $(\mathscr{I})$ et perpendiculaire $(\perp)$ au débit moyen.

Une première contribution à la dispersion qualifiée de "mécanique " ou " géométrique " est due à la distribution des lignes de courant qui conduit à une séparation de deux particules initialement très voisines. Celle-ci se produit naturellement à cause des irrégularités du champ de vitesse à l'échelle $l$ de la taille des pores. En fait, on peut penser que même dans un réseau régulier, en régime d'écoulement stationnaire, par un effet d'amplification d'erreur caractéristique du comportement chaotique de systèmes déterministes, on puisse trouver une telle dispersion au moins dans un système tridimensionnel où l'incompressibilité n'empêche nullement cette " sensibilité aux conditions initiales » [3] (par contre dans un système à 2 dimensions - réseau de cylindres - il semble que le milieu poreux doive avoir une géométrie aléatoire à cause de la conservation des longueurs dans la direction perpendiculaire à l'écoulement moyen).

Cette contribution conduit à une expression de la dispersion longitudinale de la forme suivante

$$
D_{\|} \propto U^{2} \tau=U l
$$

où $\tau=l / U$ est le temps d'exploration pour une particule de l'inhomogénéité du champ de vitesse à l'échelle $l$. Ce terme est prépondérant devant la diffusion moléculaire aux grands nombres de Peclet $\left(P e=U l / D_{m}\right)$ et décrit le comportement linéaire caractéristique d'écoulements lents si on conserve un nombre $R e$ suffisamment petit pour éviter le brassage turbulent. Si on inverse un écoulement autour d'un obstacle (sphère-cylindre) $(\mathbf{U} \rightarrow-\mathbf{U})$, on constate en effet à l'aide de marqueurs en solution, une réversibilité globale de l'écoulement. Cependant, au voisinage immédiat des points de stagnation de l'obstacle, il reste des particules de traceur qui ont été échangées par diffusion moléculaire dans les zones de vitesse lente. Bien que la masse de traceur en cause soit faible (elle est proportionnelle à une surface des pores et non au volume comme pour la dispersion mécanique), cet effet ne peut être négligé lorsque la durée de l'expérience est longue devant le temps mis par le traceur pour diffuser hors de ces zones de stagnation. Cette deuxième contribution est qualifiée de dispersion " hydrodynamique " (ou de " hold up "). Saffman [2] a étudié en premier une contribution de ce type : il a considéré un poreux modélisé par un ensemble de canaux cylindriques dans lesquels on trouve un écoulement de Poiseuille. Il a obtenu une contribution non analytique à $D_{\|}$, due au fluide stagnant au contact des parois du cylindre, en $U$ Log $U$. (Aux plus faibles nombres de Peclet tel qu'il existe un mélange important par diffusion entre l'écoulement moyen et les zones stagnantes, il existe une contribution intermédiaire en $U^{2} \tau$ qui a été étudiée par Taylor et Aris [4] dans un écoulement dans un tube cylindrique). Nous allons montrer qu'une contribution en $U \log U$ peut être obtenue dans un écoulement dans un poreux granulaire tel qu'il existe une distribution de points de stagnation de l'écoulement au niveau des grains individuels.

\section{Distribution des temps de séjour autour d'une sphère.}

Nous avons calculé la distribution $E(t)$ des temps de séjour dans l'écoulement de Stokes autour d'une sphère isolée (l'écoulement dans le milieu poreux étant supposé obéir à la loi de Darcy). $E(t) \mathrm{d} t$ représente la probabilité qu'a une particule de fluide idéale d'avoir un temps de séjour compris entre $t$ et $t+\mathrm{d} t$. Nous avons calculé le temps mis par une particule de fluide pour se déplacer entre 2 plans d'équation $x= \pm l / 2$ en intégrant numériquement la solution de Stokes. 
Le rayon de la sphère est $R$, la vitesse du fluide loin de la sphère est supposée uniforme et égale à $U_{0}$ (Fig. 1).
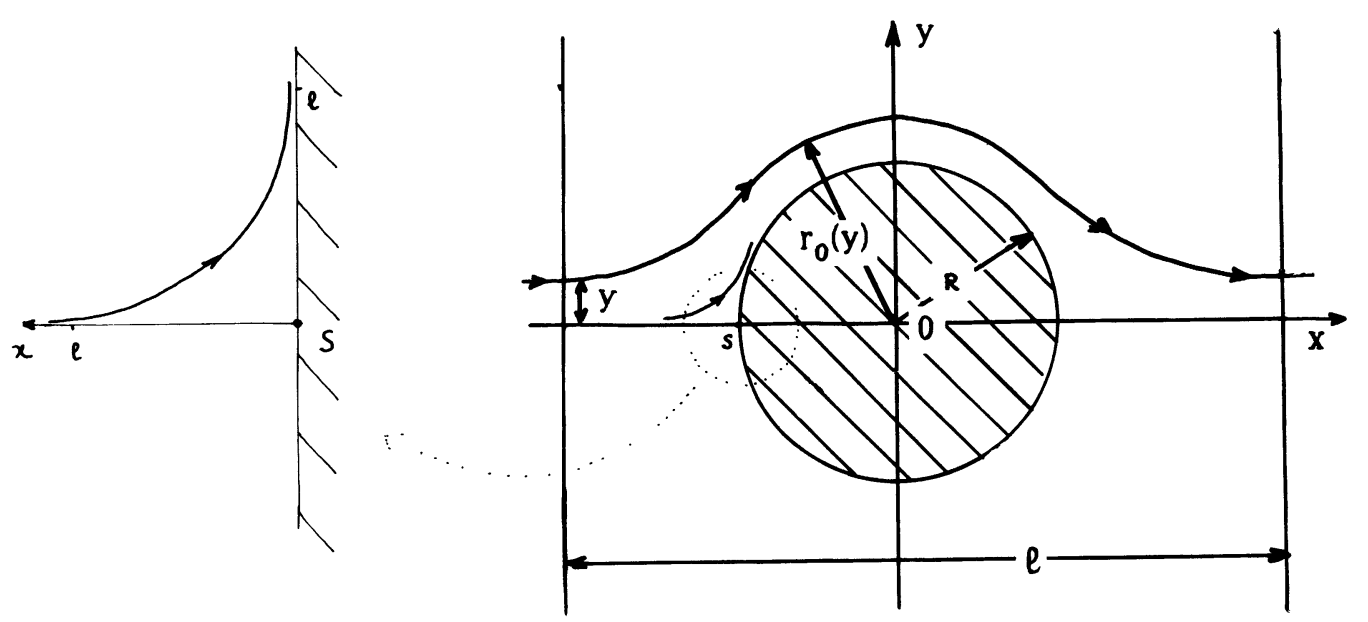

Fig. 1. - Géométrie du calcul du temps d'écoulement $t(y)$ autour d'une sphère aux faibles nombres de Reynolds entre deux plans $x= \pm l / 2$ en fonction de la distance à l'axe $x$. L'encart donne la géométrie du calcul simplifié de la contribution singulière autour du point de stagnation $S$.

[Geometry of the calculation giving the transit time $t$ between two planes $x= \pm l / 2$ along a trajectory for a flow around a sphere at low Reynolds number as a function of the distance $y$ to the axis. The inset is the geometry for the simplified calculation of the singular contribution due to the slow flow near a stagnation point S.]

Soit $t(y)$ ce temps, $y$ repérant la position de départ de la particule dans le plan d'abscisse $x=-l / 2$ :

$$
t(y)=2 \int_{\left(y^{2}+l^{2}\right)^{1 / 2}}^{r_{0}(y)} \frac{\mathrm{d} r}{u_{r}}
$$

Le graphe de cette fonction est donné sur la figure 2 où l'on distingue deux régimes : pour les grandes valeurs de $y$, le temps constant $t_{1}(y)=l / u_{0}$ correspond au transport (en ligne droite) peu perturbé par la sphère. Quand les lignes de courant se rapprochent de la sphère, on observe un raccordement net avec une loi en $y^{-1}$ que nous pouvons mettre sous la forme :

$$
t_{2}(y)=\left(R^{2} / u_{0}\right) y^{-1}
$$

et qui décrit la variation des temps de séjour pour des trajectoires très proches de la sphère (la loi est indépendante de $l$ lorsque le temps $t_{2}$ est suffisamment plus long que $t_{1}$ ).

En supposant la concentration des particules de traceur uniforme dans le plan $x=-l / 2$ $(E(t) \mathrm{d} t=y \mathrm{~d} y)$ nous obtenons pour la distribution des probabilités de temps de séjour, $E(t)$, la forme suivante (Fig. 3) :

$$
E(t)=A \delta\left(t-l / u_{0}\right)+B H\left(t-l / u_{0}\right) t^{-3} .
$$




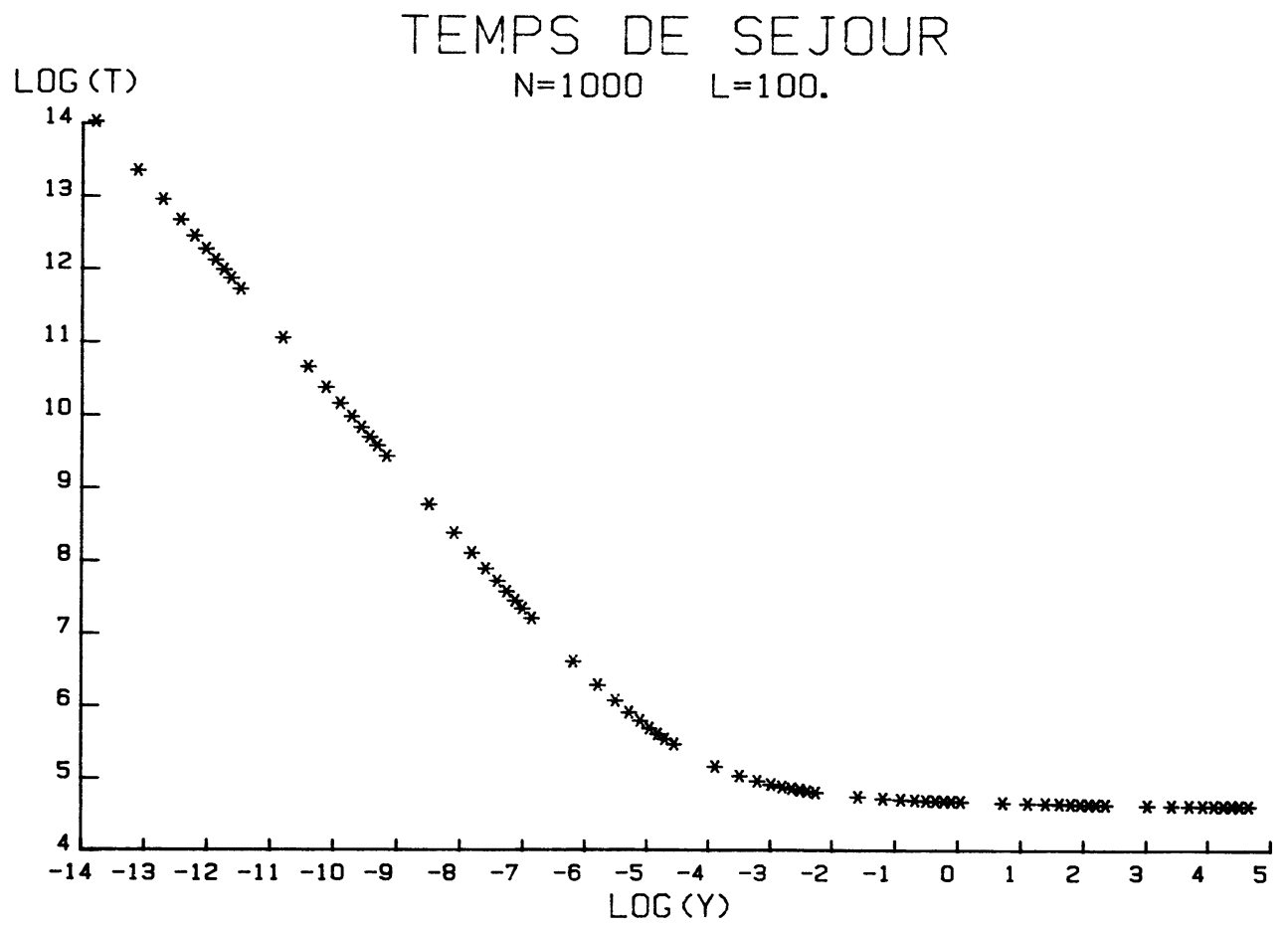

Fig. 2. - Résultat de l'intégration numérique du calcul du temps $t$ en fonction de la distance à l'axe, $y$, en coordonnées log-log. On distingue le régime singulier en $y^{-1}$ dû à l'effet des zones de stagnation pour les trajectoires près de l'axe et l'asymptote $t_{1}(y)=l / u_{0}$.

[Result of the numerical integration of the calculation of time $t$ as a function of the distance $y$ to the symmetry axis $x$ in log-log units. One gets 2 regimes : one in $y^{-1}$ due to flow lines near the axis strongly influenced by the stagnation zones; another one for farther lines, $t_{1}(y)=l / u_{0}$.]

$A$ et $B$ sont des fonctions connues de $R$ et $l: H$ est la fonction de Heaviside, $\delta$ celle de Dirac. Le-temps de résidence moyen :

$$
t=\int_{0}^{\infty} t E(t) \mathrm{d} t=A l / u_{0}+B u_{0} / l
$$

est fini et se réduit à $l / u_{0}$ pour des grands $l$ (ou des grandes séparations entre sphères).

Par contre, le second moment de la distribution des temps de séjour diverge du fait du comportement de $E(t)$ en $t^{-3}$ pour les grands temps (particule de traceur au voisinage du point de stagnation). On peut pallier cette difficulté en prenant en compte l'effet de la diffusion moléculaire qui introduit un temps de coupure inférieur $t_{\mathrm{c}}$. Le temps peut être évalué en égalant le temps de convection $t_{2}=R^{2} / u_{0} y$ et le temps de diffusion transverse à l'écoulement $t_{\text {diff }}$ sur une distance $y$ (notons que la distance minimale d'approche d'une ligne de courant donnée à la sphère est proportionnelle à la distance $y$ à l'axe loin de la sphère, soit $\left.t_{\mathrm{diff}}=y^{2} / D_{\mathrm{m}}\right)$. On trouve, en exprimant $t_{2}=t_{\mathrm{diff}}, t_{\mathrm{c}}=\left(R / u_{0}\right) P e^{1 / 3}$, d'où une dispersion carrée moyenne sur les temps de transit entre les plans $-l$ et $+l$

$$
\sigma_{t}=\overline{t^{2}}-\bar{t}^{2}=A\left\{\log \left(\frac{R}{l} P e^{1 / 3}\right)+\frac{1}{2}\right\} \text {. }
$$




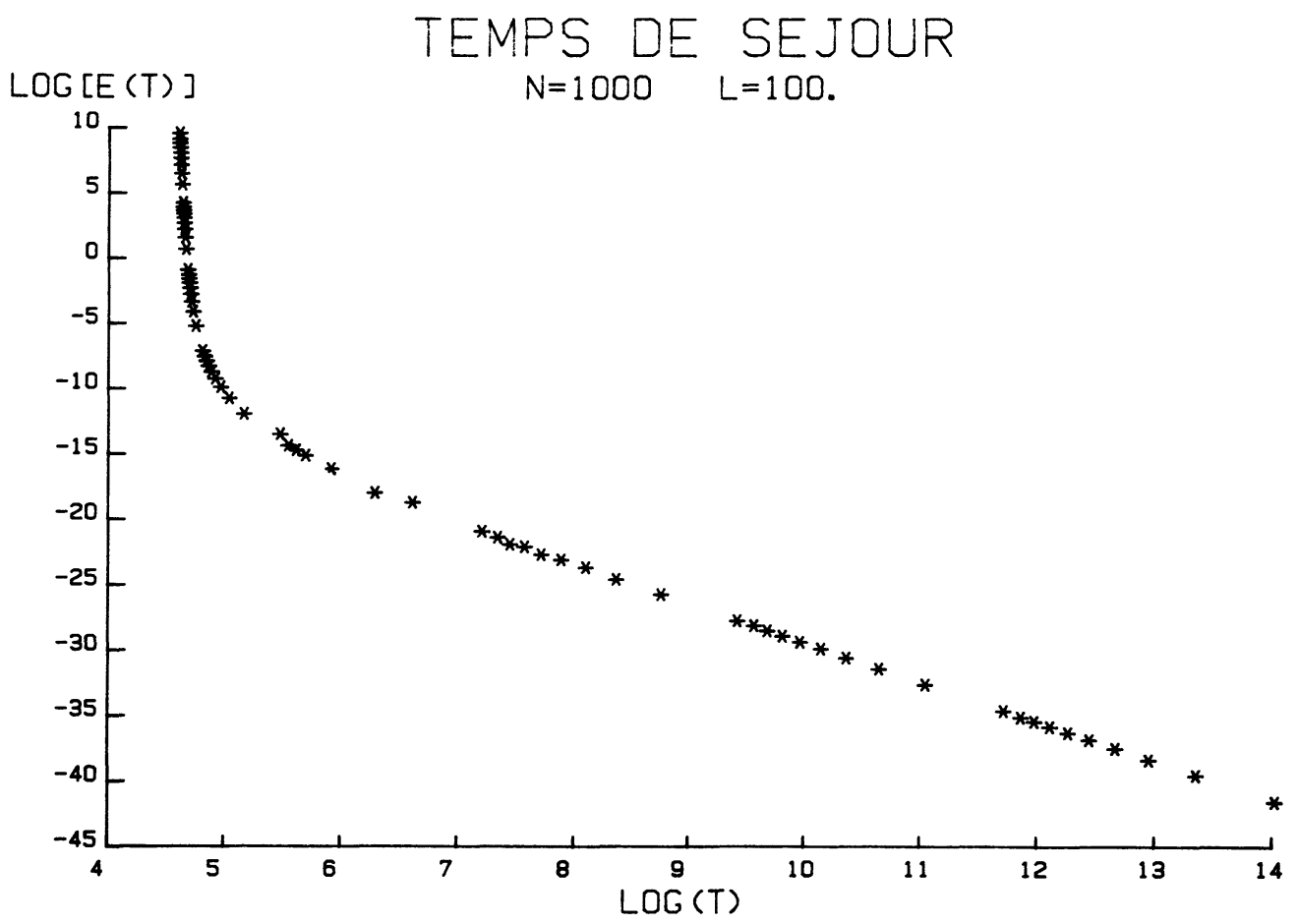

Fig. 3. - Calcul numérique de la distribution des temps de séjour $E(t)$. La divergence de $E(t)$ en $t^{-3}$ pour les temps longs est la cause de celle du coefficient de dispersion nécessitant la prise en compte d'une coupure par la diffusion moléculaire.

$[E(t) \mathrm{d} t$ is the probability that a given particle takes a time between $t$ and $t+\mathrm{d} t$ to go from $-l / 2$ to $+l / 2$. The divergence of $E(t)$ in $t^{-3}$ for long times causes the divergence of the second moment of the distribution and of the longitudinal dispersion coefficient. This singularity is removed by taking into account the long time cut off by molecular diffusion.]

Cette expression suppose que le temps de mélange $t_{\mathrm{c}}=\left(R / u_{0}\right) P e^{1 / 3}$ est assez long devant le temps convectif $t_{1}=l / u_{0}$.

Ce résultat conduit en suivant le calcul de Saffman qui prend en compte la distribution des temps de séjour due à la dispersion mécanique (dans la limite où le théorème de limite centrale s'applique) à

$$
D_{\|} \propto u_{0} l\left\{\log \left(\frac{R}{e} P e^{1 / 3}\right)+\frac{1}{2}\right\}
$$

qui corrige la formule (2) en tenant compte de l'effet de retenue autour des points de stagnation. Il est intéressant de noter que le comportement logarithmique trouvé ici et dans le problème de Saffman provient d'une singularité en $t^{-3}$ aux temps longs. La singularité faible obtenue dans les deux problèmes provient d'une certaine compensation entre la divergence en $1 / l \mathrm{du}$ temps de séjour, et le fait que le nombre de particules dont le temps de séjour est supérieur à $t_{0}$ tend vers 0 comme $1 / t_{0}$. On peut retrouver la structure en $t^{-3}$ en considérant simplement le comportement autour d'un point de stagnation sans avoir à prendre en compte la forme détaillée des obstacles comme le montre le raisonnement simplifié suivant. 


\section{Distribution des temps de séjour au voissinage d'un point de stagnation.}

Considérons l'écoulement axisymétrique au voisinage immédiat d'un point de stagnation (encart de Fig. 1). Cet écoulement peut être décrit par une fonction de courant $\psi=-k x^{2} y^{2}$.

D'où

$$
\begin{aligned}
& u=-2 k x^{2} \\
& v=2 k x y .
\end{aligned}
$$

Calculons le temps mis par une particule pour parcourir sa trajectoire entre les points $z(x=l$, $\left.y=y_{0}\right)$ et $\left(x=y_{0}, y=l\right)$ soit $t(y)$ :

$$
t\left(y_{0}\right)=\oint_{y_{0}}^{l} \frac{\mathrm{d} r}{v} \quad t\left(y_{0}\right)=\frac{1}{2 k y_{0} l}\left(l-y_{0}\right) .
$$

D’où

$$
t\left(y_{0}\right) \propto \frac{1}{2 k y_{0}} \text { lorsque } t \rightarrow \infty .
$$

Soit $E(t) \propto 1 / t^{3}$, d'après $E(t) \mathrm{d} t \propto y_{0} \mathrm{~d} y_{0}$. On retrouve donc dans le cas plus général de l'écoulement au voisinage d'une paroi, le comportement $E(t) \propto 1 / t^{3}$. Ce calcul peut être généralisé au cas où la ligne de courant arrivant ou partant du point de stagnation est oblique par rapport au plan, ce qui ne change pas la singularité décrite ici.

\section{Passage au cas de poreux de structure quelconque.}

Si on veut traiter rigoureusement le cas d'un poreux quelconque, ce qui ne présente d'ailleurs pas obligatoirement un très grand intérêt, on est conduit à un problème d'une complication redoutable, parce qu'il faut connaître en détail la structure d'un écoulement visqueux incompressible dans une géométrie quelconque. Nous allons donc nous contenter de considérations heuristiques.

Comme nous l'avons vu, la dispersion aux temps longs due à la présence d'un obstacle sphérique isolé provient de l'écoulement au voisinage du point de stagnation. Il paraît donc raisonnable de supposer que, dans un poreux de structure aléatoire mais homogène à grande échelle, constitué de sphères séparées entre elles (modèle de lit fixe) [4], il va continuer à en être de même. Nous allons caractériser le poreux par une échelle de longueur unique $R$ que l'on peut voir par exemple comme la taille moyenne des pores. Sur la surface de ce poreux, il y a une densité numérique uniforme d'ordre $R^{-2}$, de points de stagnation de l'écoulement. Ces points de stagnation sont répartis au hasard sur des lignes de courant sortantes et entrantes. D'autre part, il n'y a aucune raison pour qu'une ligne de courant issue d'un point de stagnation entrant et restant donc près de la surface du poreux émerge juste en un point de stagnation sortant. Ceci conduit donc à une estimation de $D_{\|}$analogue à la formule donnée plus haut, mais où l'on remplace $l$ par $R$. Par contre, il apparaît que, pour un poreux de taille finie, on peut avoir à modifier cette loi. En effet, on peut considérer que l'argument de Logarithme de la loi (5) est de la forme $\left(R / r_{\mathrm{D}}\right)$, où $r_{\mathrm{D}}$ est la distance de plus courte approche au solide permise par la diffusion moléculaire, soit $r_{\mathrm{D}}=\left(D R^{2} / u_{0}\right)^{1 / 3}$.

Mais il peut se faire que - pratiquement - cette distance soit si courte qu'une particule diffusante quelconque n'approche jamais de si près la paroi du solide, parce que sa trajectoire dans le poreux n'est pas assez longue. Soit donc $L$ la longueur de poreux traversée par l'écoulement. Le nombre de fois où la particule passera à une distance d'ordre $R$ d'un point de stagnation est de l'ordre de $(L / R)$. Il y a donc une densité numérique (par unité de surface) en $n_{\mathrm{c}}=L / R^{3}$ 
— d' « impacts » de la trajectoire - au voisinage des points de stagnation. Donc la distance de plus faible approche d'un point de stagnation est d'ordre $n_{\mathrm{c}}^{-1 / 2}=R(R / L)^{1 / 2}=r(L)$.

Si $r(L) \gg r_{\mathrm{D}}$ c'est la longueur finie de l'échantillon qui limitera la divergence logarithmique de l'intégrale (3) et on aura donc

$$
D_{\|} \sim u_{0} l \log |R / r(L)| .
$$

Par contre dans la limite opposée $r(L) \ll r_{\mathrm{D}}$, c'est la diffusion moléculaire qui limitera cette approche et on aura bien (5) avec $l=R$.

Remarquons aussi que, dans la situation $r(L) \gg r_{\mathrm{D}}$, la dispersion va garder une phénoménologie sensiblement différente d'une diffusion ordinaire. En effet, les particules s'étant rapprochées le plus d'un point de stagnation mettront nettement plus de temps que les autres à travers le milieu. Un pic de densité initiale s'étalera donc de façon très asymétrique, avec une queue vers les longs temps de transit. Au contraire, si $r(L) \ll r_{\mathrm{D}}$, toutes les particules se seront approchées de nombreuses fois à une distance d'ordre $r_{\mathrm{D}}$ de la paroi solide et on s'attend à ce que, dans ce cas, la tache de dispersion soit beaucoup plus proche d'une tache de diffusion gaussienne au sens ordinaire.

Dans cette étude nous n'avons pas considéré les effets de dispersion géométrique provenant de la masse retenue à l'intérieur de particules (microporeuses) ou dans des zones réellement stagnantes (comme celles présentes entre deux sphères en contact. Ce problème a été étudié sur des lits fixes par Koch et Brady [5] qui ont fait en particulier une étude des termes en $U^{2}$ (ou en $\mathrm{Pe}^{2}$ ) par opposition aux termes linéaires. Cependant comme ces auteurs le dégagent bien, ces contributions apparaissent dans la limite des temps très longs où existe un échange suffisant entre le fluide piégé et le fluide convecté.

\section{Remerciements.}

Nous avons eu de nombreuses discussions sur ce sujet avec J. Brady, E. Charlaix, J. P. Hulin. Ce travail fait partie d'un effort commun du " groupe poreux P.C. ». Nous remercions Mr. P. Lallemand, responsable du Greco expérimentation numérique pour l'accès au calculateur FPS 164 de l'E.N.S. qu'il nous a généreusement accordé.

Note ajoutée aux épreuves: Après que cet article ait été soumis, nous avons eu connaissance de l'article publié en juin 1985 au J.F.M. par Donald L. Koch et John F. Brady (J. Fluid Mech. 154 (1985) 399) qui discute en adoptant une approche différente de la nôtre le comportement logarithmique du coefficient de dispersion dû à l'existence d'un point de stagnation.

\section{Bibliographie}

[1] Fried, J. et Combarnous, M. A., Adv. Hydrosci. 7 (1971) 169.

[2] Saffman, P. G., J. Fluid Mech. 6 (1959) 321.

[3] Le modèle du billard stochastique illustre bien cette sensibilité aux conditions initiales de systèmes mécaniques à nombre de degrés de liberté suffisant (BERry, M., Eur. J. Phys. 2 (1981) 91). Dans le cas présent, l'incompressibilité de l'écoulement $3 \mathrm{D}$ joue un rôle analogue au théorème de Liouville pour la conservation des aires. Les lignes de flot d'un écoulement $3 \mathrm{D}$ autour d'obstacles disposés périodiquement vont donc avoir une structure analogue à celle d'un flot hamiltonien à 2 degrés de liberté (comme par exemple l'hamiltonien de Henon-Heiles, Astron. J. 69 (1964) 
73. On peut imaginer que l'évolution d'une particule se déplaçant le long des lignes de courant se divisera, suivant les conditions initiales, entre une dynamique quasi périodique (tores de Kam) et une dynamique chaotique (avec sensibilité aux conditions initiales). Il est toutefois imaginable que, dans certains cas, comme dans le cas des billards convexes en théorie des systèmes dynamiques, il n'y ait pas du tout de tore de Kam.

[4] Taylor, G. I., Proc. Roy. Soc. A 219 (1953) 186; ibid. 225 (1954) 473.

ArIS, R., Proc. Roy. Soc. A 235 (1956) 67.

[5] Voir Koch, D. L. et BRADY, J. F., J. Fluid Mech., à paraître. 